\title{
Equivalence of 'non-IgG binding' and 'acrosomeless' sperm populations from the female genital tract of the rabbit
}

\author{
N. J. Taylor
}

Department of Zoology and Comparative Physiology, University of Birmingham, P.O. Box 363, Birmingham B15 2TT, U.K.

\begin{abstract}
Summary. Spermatozoa were recovered from the ejaculate, vagina, uteri and oviducts of mated does between 2.5 and $14 \mathrm{~h}$ p.c. For each sample, the proportion of spermatozoa exhibiting no binding of $\mathrm{IgG}$ from normal serum, after air drying and acetone fixation, was compared with the proportion of acrosomeless spermatozoa as assessed by staining with eosin and fast green. The correlation was excellent $(r=$ $0.97 ; P<0.001)$ suggesting that failure of acetone-fixed spermatozoa to bind IgG from normal serum may reflect acrosome absence rather than 'acrosomal uncoatibility'. Usually the proportion of immunofluorescence negative or acrosomeless spermatozoa was about $10 \%$ in the ejaculate; $15 \%$ in the vagina; $25 \%$ in the uterus and $70-100 \%$ in the oviducts. This phenomenon is not an air-drying artefact, and seems to be independent of flushing medium.
\end{abstract}

\section{Introduction}

Various authors, including Allen \& Bourne (1978), Beck, Edwards \& Young (1963) and Cohen \& Werrett (1975) have studied the coating of the acrosomal region by IgG from normal serum. Allen \& Bourne (1978) showed that normal serum IgG can bind by its Fc region to the acrosomes of fixed ejaculated spermatozoa, and Cohen (1978) has further proposed that this 'non-specific' binding is selective.

Symons (1967) showed that 'senescent' spermatozoa bound IgG, and Cohen \& Tyler (1980) observed that spermatozoa reaching the site of fertilization are different from the vast majority of those in the ejaculate, in that they do not bind IgG even after air drying and acetone fixation, and have seen this as evidence of an immunological mechanism of sperm selection in the female genital tract. Cohen \& McNaughton (1974) showed that oviducal spermatozoa were a very fertile, presumably selected, population, as are spermatozoa that arrive early in the uterus and are immunofluorescence negative (Cohen \& Tyler, 1980).

Because it seemed likely that acrosome loss (Bedford, 1970) and acrosomal IgG-binding properties of spermatozoa were related, the present paper investigated whether spermatozoa that had lost their acrosomes were also unable to bind IgG.

\section{Materials and Methods}

Ejaculated spermatozoa were collected from one buck of proven fertility by artificial vagina and teaser doe. Samples were observed undiluted by phase-contrast microscopy, and the proportion of spermatozoa 'without acrosomes' was recorded (3 assessments of 100 spermatozoa per 
sample). Spermatozoa from the same ejaculate were suspended in phosphate-buffered saline (PBS, Dulbecco A) and air-dried on microscope slides from 'deep' (2 mm) or 'shallow' $(0.1 \mathrm{~mm})$ drops at 20 or $60^{\circ} \mathrm{C}$. The slides were then smeared with Wells \& Awa (1970) stain (1 part 1\% aqueous eosin B; 2 parts $1 \%$ aqueous fast green F.C.F.; 2.7 parts ethanol), left for 1 min and air-dried at $60^{\circ} \mathrm{C}$. The proportion of 'acrosomeless spermatozoa' after each drying step was assessed ( 3 assessments of 100 spermatozoa per sample) by bright-field microscopy because phase-contrast did not satisfactorily differentiate the colours.

The buck was allowed to mate once with each of 4 parous Dutch does. At $2 \cdot 5,6,10 \cdot 5$ or 14 h post coitum (p.c.) one doe was anaesthetized by intravenous pentabarbitone sodium and exsanguinated by cardiac puncture on a warm operating table. Within $1 \mathrm{~min}$ of death the abdomen had been opened and the genital tract clamped with haemostats at the utero-tubal junctions and $1 \mathrm{~cm}$ above and $2 \mathrm{~cm}$ below the cervix. The reproductive tract was flushed by the method of Cohen \& Werrett (1975) with warm PBS (0.5 ml per oviduct, $1 \mathrm{ml}$ per uterine horn or vagina). A sample $(20 \mu \mathrm{l})$ of each flush was observed directly by phase-contrast microscopy, while the remainder of each flush was suspended in $10 \mathrm{ml}$ PBS, centrifuged at $400 \mathrm{~g}$ for $10 \mathrm{~min}$ and finally resuspended in a small volume of fresh PBS $(0.05 \mathrm{ml})$. Ejaculated spermatozoa from the same buck were similarly washed 3 times in fresh PBS.

Half of each sample was then air-dried and stained with eosin-fast green and the proportions of 'acrosomeless spermatozoa' were assessed as before; in oviducal samples all spermatozoa recovered were assessed.

The remainder was prepared for direct immunofluorescence staining. Samples from each flush were air-dried onto chemically clean microscope slides, previously diamond marked to give 11 areas. Areas 1 and 2 contained thrice-washed ejaculated spermatozoa, areas 3 and 4 vaginal spermatozoa, areas 5 and 6 uterine spermatozoa, areas 7, 8,9 and 10 oviducal spermatozoa, and area 11 rabbit serum diluted $1 / 100$ in PBS as a control for IgG visualization. Thus, on each slide there were some spermatozoa expected to stain immunofluorescence positive and some negative. After drying the slides were fixed in dry acetone (over anhydrous sodium sulphate) for $30 \mathrm{~min}$ at $-20^{\circ} \mathrm{C}$ and then washed 10 times in $250 \mathrm{ml}$ PBS per wash, over $1 \mathrm{~h}$, to remove the acetone.

Immunofluorescence staining was performed by treating the air-dried, fixed and washed spermatozoa with undiluted normal rabbit serum for $30 \mathrm{~min}$. After washing, IgG on spermatozoa was visualized with fluorescein-labelled sheep anti-rabbit IgG (Wellcome MF 02; Wellcome Reagents Ltd, Hithergreen, London, U.K.). After treatment for $30 \mathrm{~min}$, the slides were washed in PBS and mounted with U-Vinert (Gurr) and observed by simultaneous incident-light fluorescence and red phase-contrast to discriminate immunofluorescence-negative spermatozoa. The proportion of immunofluorescence-negative spermatozoa in each sample was assessed (3 assessments of 100 spermatozoa per sample; all spermatozoa recovered in oviducal samples).

To compare PBS with a more 'complex' flushing medium, spermatozoa were recovered from the 2 uterine horns of a doe at $2 \mathrm{~h}$ p.c. using PBS for one horn and the medium described by Brackett \& Oliphant (1975) for the other. The proportion of 'acrosomeless spermatozoa' in each sample was assessed by staining with eosin-fast green as above.

\section{Results}

Air drying and staining with eosin-fast green distinguishes rabbit spermatozoa with acrosomes (purple head, green acrosome), from acrosomeless spermatozoa (bright pink head). The proportions of spermatozoa assessed as 'acrosomeless' while in the ejaculate, or after suspension in PBS followed by various air-drying steps (Table 1$)$ were the same $(P<0.05$, Student's $t$-test).

There was no significant difference ( $t$ test) in the proportions of spermatozoa assessed as 'acrosomeless' in the two uterine horns of a doe when PBS $(12 \pm 2 \%)$ or Brackett \& Oliphant's 
(1975) medium $(13.3 \pm 3 \%$ ) were used (mean \pm s.d. of 6 assessments of 100 different spermatozoa per assessment).

Table 1. Proportions (\%) of rabbit spermatozoa assessed as 'acrosomeless', by eosin-fast green staining, in ejaculated spermatozoa suspended in PBS, followed by various air-drying steps, and in untreated live ejaculated spermatozoa as assessed by phase-contrast microscopy

\begin{tabular}{lc}
\hline \multicolumn{1}{c}{ Drying process } & Acrosomeless spermatozoa (\%) \\
\hline Deep drop at $20^{\circ} \mathrm{C}$ & $12 \cdot 3 \pm 0.5$ \\
Deep drop at $60^{\circ} \mathrm{C}$ & $11 \cdot 3 \pm 0.5$ \\
Shallow drop at $20^{\circ} \mathrm{C}$ & $11 \cdot 3 \pm 0.5$ \\
Shallow drop at $60^{\circ} \mathrm{C}$ & $12 \cdot 3 \pm 0.5$ \\
Ejaculated (live) & $12.5 \pm 3.5$ \\
\hline
\end{tabular}

Values are mean \pm s.d. for 600 spermatozoa, using 100 spermatozoa at each assessment.

The proportions of acrosomeless spermatozoa varied (Table 2), the values increasing with ascent through the female reproductive tract: in the oviduct $\geqslant 75 \%$ of spermatozoa had lost their acrosomes. The correlation between the proportions of spermatozoa assessed as 'acrosomeless' and those that did not fluoresce, from all regions of the tract, was excellent (Pearson's Correlation Coefficient; $r=0.97, P<0.001$ ).

Table 2. Proportions (\%) of rabbit spermatozoa that were immunofluorescence (IF)-negative or without an acrosome (eosin-fast green stain: E-FG) in different parts of the female tract from 4 does ( 1 killed at each time)

\begin{tabular}{|c|c|c|c|c|c|}
\hline \multicolumn{2}{|c|}{ Sample } & \multicolumn{4}{|c|}{$\%$ IF negative or acrosomeless } \\
\hline h p.c. & Method & Ejaculate & Vagina & Uterus & Oviduct \\
\hline $2 \cdot 5$ & $\begin{array}{l}\text { IF } \\
\text { E-FG } \\
\text { Live* }\end{array}$ & $\begin{array}{r}9.2 \pm 2.3 \\
10.5 \pm 2.4 \\
+\end{array}$ & $\begin{array}{r}9.2 \pm 2.3 \\
15.7 \pm 3.8 \\
+\end{array}$ & $\begin{array}{c}16.0 \pm 3.8 \\
19.2 \pm 5.6 \\
++\end{array}$ & $\begin{array}{c}84.0 \pm 12 \cdot 8 \\
100 \cdot 0(7 / 7) \\
+++++\end{array}$ \\
\hline $6 \cdot 0$ & $\begin{array}{l}\text { IF } \\
\text { E-FG } \\
\text { Live* }\end{array}$ & $\begin{array}{c}10 \cdot 5 \pm 1 \cdot 4 \\
11 \cdot 3 \pm 2 \cdot 4 \\
+\end{array}$ & $\begin{array}{c}19.8 \pm 3.1 \\
23.3 \pm 2.8 \\
++\end{array}$ & $\begin{array}{c}15.7 \pm 2.7 \\
17.8 \pm 2.2 \\
+\end{array}$ & $\begin{array}{c}84.8 \pm 5.4 \\
75.0 \pm 9.6 \\
+++++\end{array}$ \\
\hline $10 \cdot 5$ & $\begin{array}{l}\text { IF } \\
\text { E-FG } \\
\text { Live* }\end{array}$ & $\begin{array}{c}12.8 \pm 2.2 \\
12.8 \pm 0.9 \\
+\end{array}$ & $\begin{array}{c}15.8 \pm 2.5 \\
15.8 \pm 1.9 \\
+\end{array}$ & $\begin{array}{c}23.0 \pm 2.6 \\
30.0 \pm 3.4 \\
++\end{array}$ & $\begin{array}{c}93.8 \pm 7.5 \\
74.2 \pm 6.3 \\
+++++\end{array}$ \\
\hline 14 & $\begin{array}{l}\text { IF } \\
\text { E-FG } \\
\text { Live* }\end{array}$ & $\begin{array}{c}12.5 \pm 3.0 \\
16.5 \pm 2.9 \\
+\end{array}$ & $\begin{array}{c}-t \\
29.6 \pm 3.9 \\
++\end{array}$ & $\begin{array}{c}25 \cdot 5 \pm 3 \cdot 3 \\
24 \cdot 8 \pm 5 \cdot 3 \\
++\end{array}$ & $\begin{array}{l}99.0 \pm 1.5 \\
80.0(20 / 25) \\
+++++\end{array}$ \\
\hline
\end{tabular}

Values are mean \pm s.d. for 6 assessments; 100 spermatozoa per assessment from each sample, except for oviducal spermatozoa for which 50 spermatozoa were scored per assessment, only 300 per sample. When no s.d. is given, $<300$ spermatozoa were recovered (number shown).

+ Not recorded; the 14-h vaginal sample had very high background fluorescence and no assessments were made.

* Assessments were made of acrosomeless live spermatozoa in each sample by phasecontrast microscopy:,$+<20 \% ;++, 20-40 \% ;+++, 40-60 \% ;++++, 60-80 \%$; ,$++++>80 \%$. 


\section{Discussion}

The staining of the rabbit acrosome by eosin-fast green is very clear and repeatable, and was the same in each sample after various air-drying steps and flushing or drying-down media. The observations of similar proportions of acrosomeless spermatozoa among the living spermatozoa, before washing, discounts the possibility of damage caused by centrifugation.

The immunofluorescence results obtained, i.e. with reduced proportions of spermatozoa staining positively as the spermatozoa ascend the reproductive tract, are in good agreement with those of Cohen \& Werrett (1975), Tyler (1977) and Cohen \& Tyler (1980). Because of the agreement of the present results with those of previously published work, it was not considered necessary to use larger numbers of animals in the present experiments, and it is probable that immunofluorescence-negative spermatozoa are acrosomeless. Cohen \& Gregson (1978) reported immunofluorescence-negative spermatozoa with acrosomes, and it is possible that a few spermatozoa with acrosomes are immunofluorescence-negative.

Sperm transfer experiments (Cohen \& McNaughton, 1974; Cohen \& Tyler, 1980) have shown that populations of immunofluorescence-negative spermatozoa are very fertile (100-150 of such spermatozoa are needed for 1 spermatozoon to fertilize an egg, compared with the $10^{4}-10^{5}$ washed ejaculated spermatozoa needed in the same system). These results have been used as evidence for a theory of sperm selection by the female tract on an immunological basis. If acrosomes are lost from spermatozoa as they ascend the tract (see "false acrosome reactions": Bedford, 1970), then recruitment of immunofluorescence-negative from immunofluorescencepositive populations would consist only of conversion of spermatozoa; this is not selection of an original sub-population in the ejaculate. The differences in IgG binding reported here are apparently associated with a morphological change, so this change has probably resulted in the differences seen by immunofluorescence between sperm populations reported by Cohen \& Tyler (1980) and Tyler (1977). There seems no basis for belief in immunological sperm selection in the fermale genital tract of the rabbit of the type suggested by Cohen \& Werrett (1975).

I thank Dr Jack Cohen for helpful discussions and Toxicol Laboratories Ltd for donating the experimental animals used in this study. This work was supported by an S.R.C. Studentship.

\section{References}

Allen, G.L. \& Bourne, F.J. (1978) Interaction of immunoglobulin fragments with the mammalian sperm acrosome. J. exp. Zool. 203, 271-276.

Beck, J.S., Edwards, R.G. \& Young, M.R. (1963) Immune fluorescence technique and the isoantigenicity of mammalian spermatozoa. J. Reprod. Fert. 4, 103-110.

Bedford, J.M. (1970) Sperm capacitation and fertilization in mammals. Biol. Reprod. 2, Suppl. 2, 128-158.

Bracketh, B.G. \& Oliphant, G. (1975) Capacitation of rabbit spermatozoa in vitro. Biol. Reprod. 12, 260-274.

Cohen, J. (1978) Non-specific (Fc) but selective attachment of antibody to sperms. Proc. 3rd Int. Symp. Immunology of Reproduction, pp. 234-238. Ed. K. Bratanov. Bulgarian Academy of Sciences, Sofia.

Cohen, J. \& Gregson, S. (1978) Antibodies and sperm survival in the female genital tract. In Spermatozoa, Antibodies and Infertility, pp. 17-31. Eds J. Cohen \& W. F. Hendry. Blackwell, London.
Cohen, J. \& McNaughton, D.C. (1974) Spermatozoa: the probable selection of a small population by the genital tract of the female rabbit. J. Reprod. Fert. 39, 297-310.

Cohen, J. \& Tyler, K.R. (1980) Sperm populations in the female genital tract of the rabbit. J. Reprod. Fert. 60 , 213-218.

Cohen, J. \& Werrett, D.J. (1975) Antibodies and sperm survival in the female tract of the mouse and rabbit. J. Reprod. Fert. 42, 301-310.

Symons, D.B.A. (1967) Reactions of spermatozoa with uterine and serum globulin determined by immunofluorescence. $J$ Reprod. Fert. 14, 163-165.

Tyler, K.R. (1977) Studies on the interactions between spermatozoa, immunoglobulins and the female genital tract in the rabbit and human. Ph.D. thesis, University of Birmingham.

Wells, M.E. \& Awa, O.A. (1970) A new technique for assessing acrosomal characteristics of spermatozoa. J. Dairy Sci. 53, 227-232. 